\title{
Tumor mucoepidermoide de la tráquea: reporte de caso
}

\section{Mucoepidermoid tumor of the trachea. Case report}

\author{
ENRIQUe FERRER-AÑEZ, MD ${ }^{1}$ BERnARdo SERRUTO-CONTRERAS, MD²
}

\section{Resumen}

De forma excepcional, la tráquea es asiento de tumores malignos. En el presente artículo se reporta un caso de carcinoma mucoepidermoide operado con resección y anastomosis término terminal.

Los tumores malignos de la tráquea son sumamente raros. Se estima que su incidencia es 1 caso por cada 180 de cáncer pulmonar. La mayor parte de los pacientes consulta por presentar disnea de larga data, habitualmente varios meses antes de diagnosticarse, que muy frecuentemente, y debido a la presencia de sibilancias, se confunde su diagnóstico con asma bronquial.

La variedad más común de neoplasia maligna de la tráquea es el carcinoma adenoide quístico, luego, en frecuencia, le sigue el carcinoma epidermoide y, por último, el carcinoma mucoepidermoide.

Palabras clave: tráquea, cirugía, tumor, carcinoma mucoepidermoide.

\begin{abstract}
We report one case of mucoepidermoid carcinoma, operated with resection and end to end anastomosis.

Malignant tumors of the trachea are extremely rare, with an estimated incidence of 1 for every 180 cases of lung cancer. The majority of the patients consulted for having longstanding dyspnea, usually several months before being diagnosed, and very frequently, due to the presence of wheezing, their diagnosis is confounded with bronchial asthma.
\end{abstract}

The most common variety of malignant neoplasm of the trachea is adenoid cystic carcinoma, followed in frequency by squamous cell carcinoma, and finally mucoepidermoid carcinoma.

Keywords: trachea, surgery, tumor, mucoepidermoid carcinoma.

\author{
${ }^{1}$ Médico especialista en Cirugía Torácica \\ General. Médico del Departamento de \\ Cirugía en Tórax del Instituto Nacional \\ de Enfermedades Neoplásicas; Lima, \\ Perú. \\ ${ }^{2}$ Médico especialista en Anestesiología y \\ Reanimación. Médico del Departamento \\ de Anestesia y Reanimación del Instituto \\ Nacional de Enfermedades Neoplásicas; \\ Lima, Perú. \\ Autor de correspondencia \\ Avenida San Borja Norte 1229; Dpto. \\ 602. Lima, Perú. Correo electrónico: on- \\ cologiatoraxica@gmail.com \\ Tel.: (+51) 986.405.432 \\ Recibido: 23/06/17. Aceptado: 20/09/17.
}




\section{Reporte de caso}

Paciente mujer de 61 años, natural y procedente de Cusco, Perú, quien desde hace aproximadamente 1 año presenta tos seca exigente, en aumento e intermitentemente, la cual se hace productiva y hemoptoica, además de disnea progresiva; por lo que acude al hospital de su localidad donde recibió tratamiento sintomático para broncoespasmo en repetidas ocasiones, sin lograr mejoría. Se derivó al Instituto Nacional de Enfermedades Neoplásicas donde se le hospitalizó. A su ingreso se le realizó radiografía de tórax (Figura 1), en la que se evidenció tumoración exofítica de $13 \mathrm{~mm}$ de diámetro en tercio medio de tráquea; además se llevó a cabo una escanografía de tórax, evidenciándose un tumor endobronquial en tercio medio de la tráquea, con densidad de partes blandas, polipoideo, que ocluye el $90 \%$ de la luz traqueal, y adenopatías mediastinales reactivas (Figura 2). Se programó para fibrobroncoscopia y biopsia, con resultado de carcinoma epidermoide; 2 días después se realizó resección traqueal segmentaria a través de esternotomía media, con anastomosis termino terminal. El resultado patológico definitivo correspondió a carcinoma mucoepidermoide $\mathrm{p} 63$ positivo, CK5/6 positivo, bordes de sección traqueal libres de enfermedad.

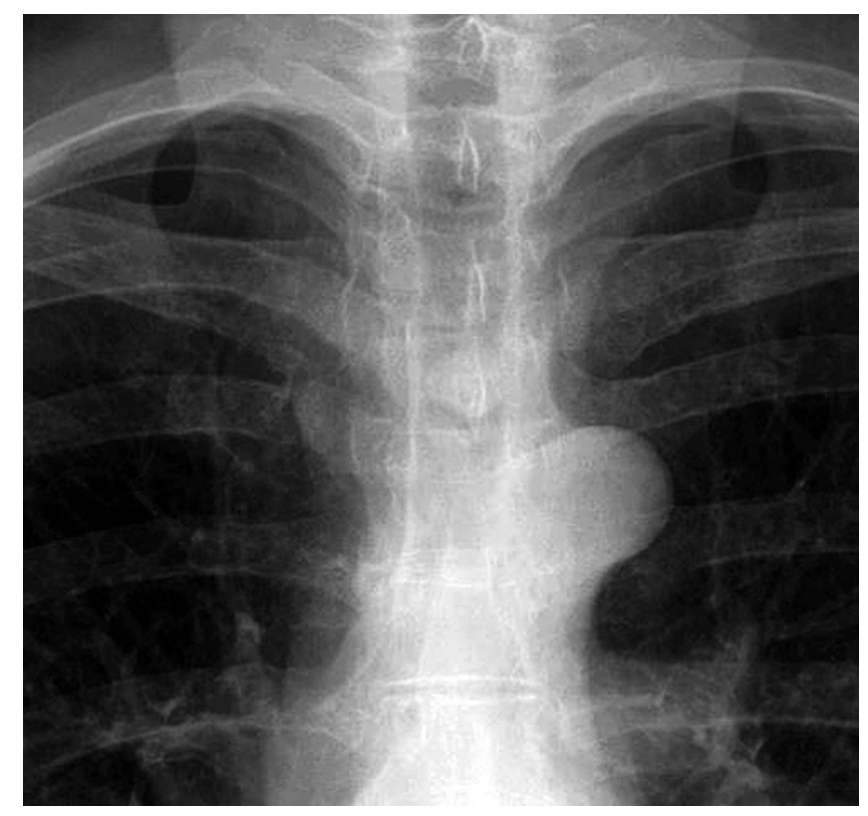

Figura 1. Detalle de la radiografía de tórax frontal al momento del ingreso. Se evidencia lesión tumoral endotraqueal, exofítica y polipoidea de $13 \mathrm{~mm}$ de diámetro, a $5 \mathrm{~cm}$ de la carina principal, que ocluye el $90 \%$ de la luz.

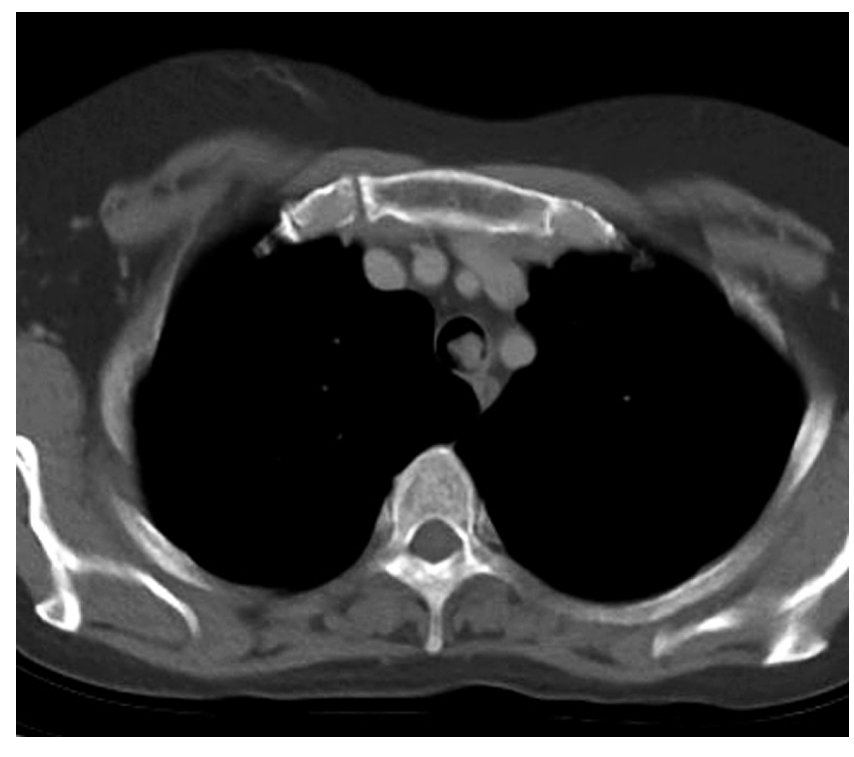

Figura 2. Tomografía espiral multicorte del tórax al momento del ingreso hospitalario. A nivel traqueal se observa lesión polipoidea de aproximadamente $13 \mathrm{~mm}$ de diámetro. No se evidencian adenopatías mediastinales groseras.

En su seguimiento posoperatorio, la paciente no tuvo complicaciones. Su último control tomográfico, 7 meses después de realizada la cirugía, no mostró recurrencia de la enfermedad (Figura 3).

\section{Discusión}

Los tumores primarios de la tráquea son sumamente raros. Debido a ello, no existen estudios de grandes series. Lo cierto es que, con base en los datos publicados, los tumores traqueales comprenden menos del $0,1 \%$ de todas las neoplasias, y por cada tumor maligno de la tráquea se producen 20 casos de cáncer de laringe y 160 a 180 de cáncer pulmonar $(1,2)$.

La enfermedad es predominante en pacientes masculinos, en una relación 7:1. Los carcinomas epidermoide, adenoide quístico y tumor carcinoide comprenden $90 \%$ de los casos. El carcinoma mucoepidermoide se relaciona con menos del $3 \%$ de los tumores traqueales primarios. El grupo etario afectado con mayor frecuencia es la población mayor de 60 años (2). En una serie de la década de 1970, 1 de cada 7 tumores fue de alto grado (3).

El tratamiento óptimo debe incluir la resección completa de la lesión; de hecho, es el único tratamiento para 


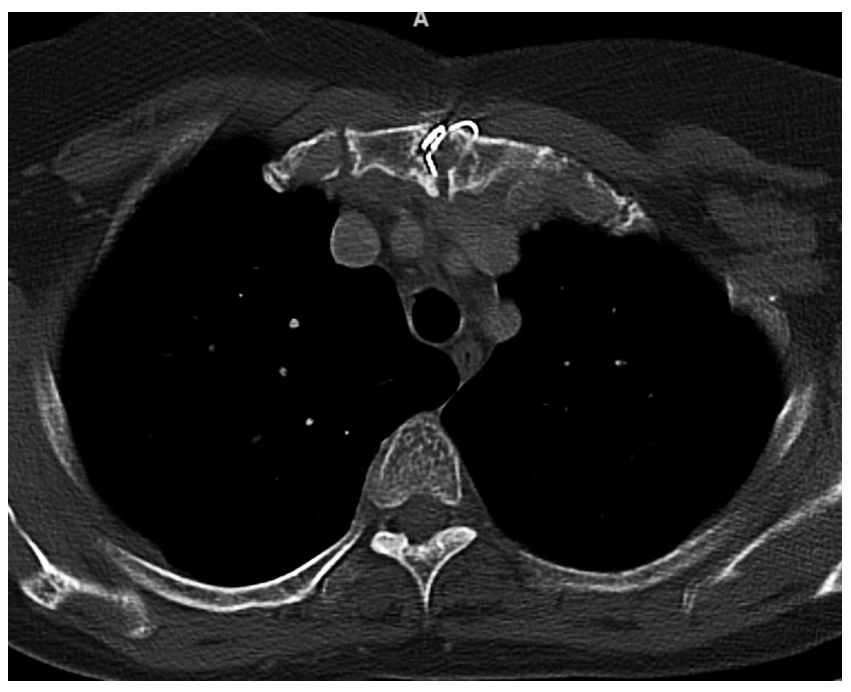

Figura 3. Tomografía espiral multicorte de tórax luego de 7 meses de la resección traqueal. Solo se evidencian cambios posoperatorios.

las lesiones de bajo grado. La endoscopia terapéutica solo se indica para mejorar la ventilación en aquellos pacientes en que esta se ve comprometida, y será suficiente obtener un cilindro de tejido con el broncoscopio rígido. Cualquier otro método de ablación con energía (argon beam, láser, electrocauterio, entre otras) solo se justifica si hay disponibilidad para ello $(4,5)$.

Las tumorectomías no constituyen un tratamiento definitivo, y el paciente siempre debe programarse para resección con bordes libres. En el caso de la tráquea, esto impone ciertas consideraciones. En primer lugar, se trata de un órgano poco elongable; segundo, no existe un sustituto artificial confiable y eficaz en el caso de resecciones amplias; y tercero, las dehiscencias en cirugía traqueal pueden ser letales, y cuando menos peligrosas, por el riesgo de trastorno severo de la ventilación y/o mediastinitis. Lo cierto es que la resección curativa amplia se debe realizar en todo paciente en el que no se ha demostrado metástasis a distancia, ni signos de invasión local. En el caso de esta paciente, los estudios de extensión fueron negativos para enfermedad secundaria, característica común en la mayoría de los tumores mucoepidermoides de la tráquea y bronquios $(2,3)$.
En cuanto a la extensión de tráquea a resecar, esto depende de la contextura, edad del paciente, tipo histológico y longitud de la lesión. Mientras en un joven, delgado, de cuello largo se puede resecar sin problemas hasta $6 \mathrm{~cm}$ de tráquea, en una persona, anciana, cuello corto, obesa y con cifosis, una resección de 4 cm implicará exceso de tensión en la anastomosis (47). En este caso, el cuello de la paciente era largo y delgado, por lo que no hubo problemas al resecar 4 anillos traqueales, lo que permitió tener bordes de sección libres de enfermedad y una anastomosis término terminal sin tensión.

Para lograr buenos resultados, otro detalle de suma importancia es el manejo anestésico. Durante la cirugía, mientras se realizaba la resección traqueal, el cabo distal se ventiló con un tubo de ánodo con manguito (Figura 4) que se fijó con seda 0 a la piel de la paciente para evitar su movilización. El tubo endotraqueal colocado transoral no se retira, sino que se mantiene justo proximal a la anastomosis para reintroducirlo una vez se complete esta y evitar hiperextensión del cuello del paciente. Para la anastomosis se utilizó ácido poliglicólico 3-0 a puntos simples transtraqueales, reforzando los ángulos laterales en la unión membranosa cartílago con puntos en "u" del mismo material. Se confirmó ausencia de fuga aérea al terminar la anastomosis (Figura 5). En el lecho operatorio se dejó el drenaje aspirativo a presión negativa, siendo retirado a las 48 horas. La paciente tuvo una evolución posoperatoria sin eventualidades, y fue dada de alta al séptimo día.

Una de las complicaciones más temidas en cirugía traqueal es la estenosis o dehiscencia de la anastomosis, lo que se relaciona con una cirugía realizada con tensión excesiva o infección del sitio operatorio con o sin dehiscencia de la anastomosis. En cirugía traqueal, la necesidad de reintervención por complicaciones quirúrgicas suele acompañarse de larga estancia hospitalaria y malos resultados finales de la reconstrucción traqueal, además de muerte por mediastinitis.

La paciente en cuestión no presentó ninguna complicación perioperatoria. Su contextura facilitó una adecuada flexión del cuello, la tráquea se movilizó en sentido craneocaudal en toda su cara anterior sin afec- 


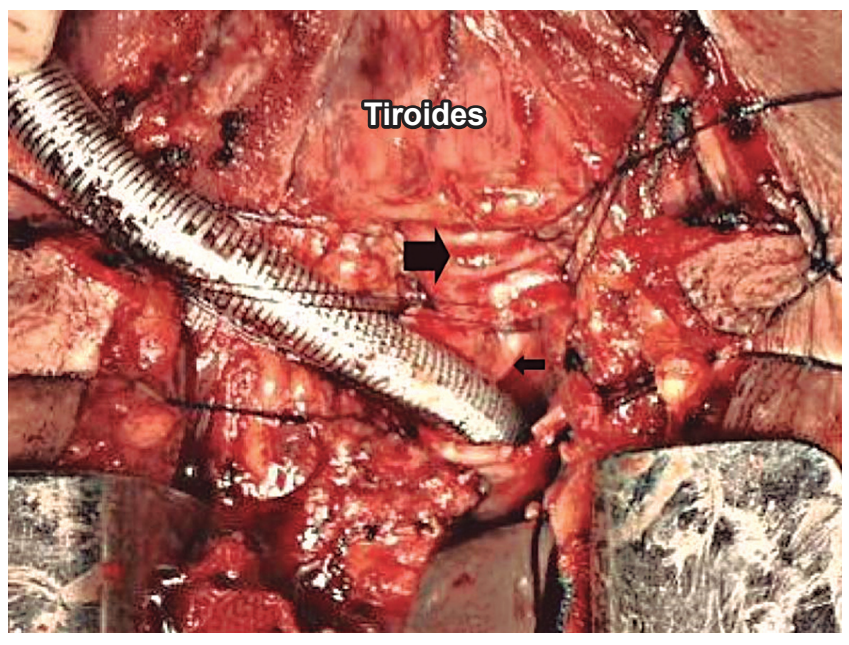

Figura 4. Detalle de la resección traqueal. Se observa el defecto dejado por el segmento de tráquea extirpada con el tubo endotraqueal anillado introducido en el cabo distal de la tráquea torácica. La flecha grande señala el extremo proximal de la tráquea; mientras que la flecha pequeña marca el extremo distal del tubo orotraqueal que se progresa hacia la tráquea distal una vez culminada la anastomosis traqueal término terminal.

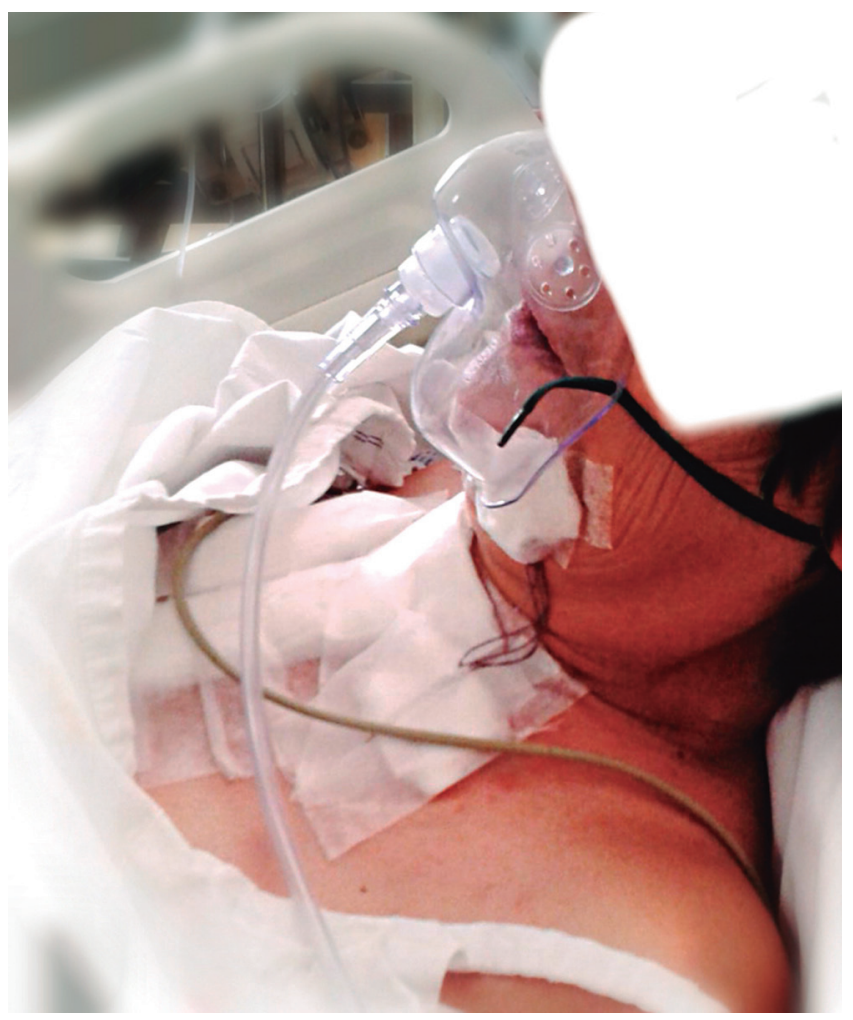

Figura 6. Detalle de la sutura de seguridad que se fija a ambos lados del mentón y la región subclavicular bilateral a fin de limitar la extensión del cuello y, de esta forma, asegurar una anastomosis traqueal libre de tensión.

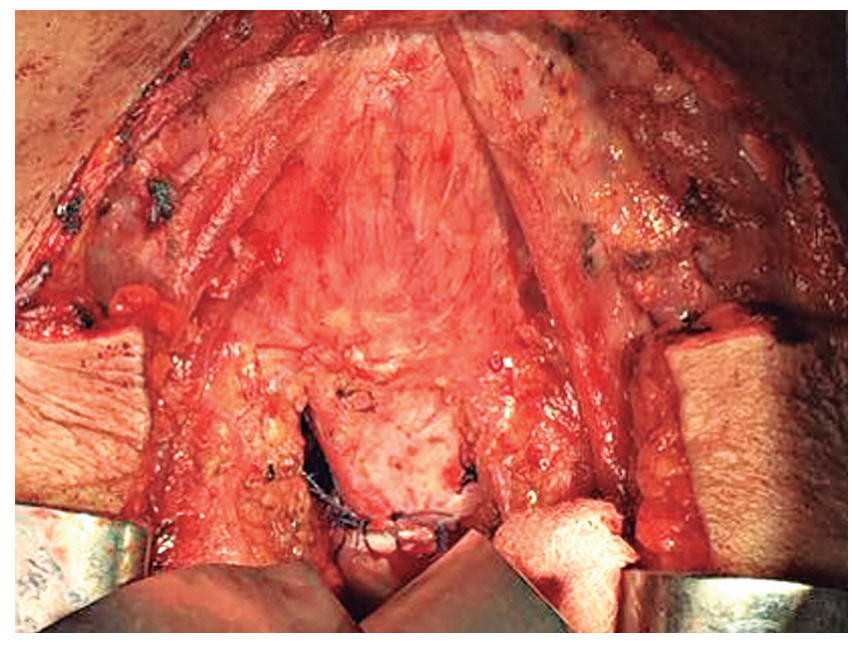

Figura 5. Se muestra la anastomosis traqueal término terminal con puntos simples de sutura absorbible de ácido poliglicólico 3-0. Se observa la disección limitada a la cara anterior de la tráquea con mínima disección lateral para preservar la irrigación del órgano.

tar las caras laterales, a fin de preservar la irrigación del órgano y evitar estrechez (Figura 5) y dehiscencia anastomótica. Terminada la cirugía se fijó el mentón a la piel sobre las clavículas con sutura absorbible polidioxanona monofilamento número 1 (Figura 6).

La radiografía de tórax no es de utilidad para el diagnóstico o seguimiento de los pacientes operados; sin embargo, en nuestra paciente fue fundamental como estudio de inicio, pues la masa tumoral se hizo evidente. La espirometría suele ser normal a pesar de la obstrucción de la vía aérea mayor (8). El seguimiento posoperatorio debe incluir escanografía de tórax y endoscopia del árbol traqueobronquial según los resultados de la tomografía o guiado por síntomas clínicos de recurrencia o que sugieran estenosis. Para las lesiones de bajo grado, la sobrevida a 1 año es del $80 \%$ y a 5 años del $57 \%$, llegando hasta $100 \%$ en centros de alta especialización en cirugía traqueal (9). El factor de peor pronóstico es una histología predominantemente epidermoide y la presencia de adenopatías mediastinales (10). Las recurrencias locales son las más comunes, siendo generalmente tratadas con reintervención y/o radioterapia. En este grupo de pacientes, la sobrevida a 1 año no es mayor del $20 \%(3,10,11)$. 


\section{Referencias}

1. Roth J, Ruckdeschel J, Weisenburger T. Thoracic oncology. $2^{\mathrm{a}}$ edición. Philadelphia: WB Saunders. 1995. p. 613.

2. Goldberg M, Patchefsky A. Uncommon tumors of the tracheobronchial tree: diagnosis and management. Chest Surg Clin N Am. 2003;13(1):1-167. DOI: 10.1016/S10523359(02)00073-X.

3. Leonardi HK, Jung-Legg Y, Legg MA, Neptune WB. Tracheobronchial mucoepidermoid carcinoma. Clinicopathological features and results of treatment. J Thorac Cardiovasc Surg. 1978;76(4):431-8.

4. Pearson G, Cooper J, Deslauriers J, Ginsberg R, Hiebert C, Patterson G, et al (editores). Thoracic surgery. 2a edición. Edinburgh: Churchill Livingstone. 2002.

5. Mathisen DJ. Tracheal surgery. Chest Surg Clin N Am. 2003;13(2):175-404. DOI: https://doi.org/10.1016/S10523359(03)00048-6.

6. Grillo HC. Surgery of the trachea. Curr Probl Surg. 1970;7(7):359. DOI: https://doi.org/10.1016/S0011-3840(70)80003-X.
7. Mathisen DJ. Surgery of the trachea. Curr Probl Surg. 1998;35(6):455-542. DOI: https://doi.org/10.1016/S00113840(98)80014-2.

8. Giusti RJ, Flores RM. Mucoepidermoid carcinoma of the bronchus presenting with a negative chest X-ray and normal pulmonary function in two teenagers: two case reports and review of the literature. Pediatr Pulmonol. 2004;37(1):81-4. DOI: $10.1002 /$ ppul.10390.

9. Gaissert HA, Grillo HC, Shadmehr MB, Wright CD, Gokhale M, Wain JC, et al. Uncommon primary tracheal tumors. Ann Thorac Surg. 2006;82(1):268-73. DOI: https://doi. org/10.1016/j.athoracsur.2006.01.065.

10. Chin CH, Huang CC, Lin MC, Chao TY, Liu SF. Prognostic factors of tracheobronchial mucoepidermoid carcinoma. 15 years' experience. Respirology. 2008;13(2):275-80. DOI: 10.1111/j.1440-1843.2007.01207.x.

11. Yang KY, Chen YM, Huang MH, Perng RP. Revisit of primary malignant neoplasms of the trachea: clinical characteristics and survival analysis. Jpn J Clin Oncol. 1997;27(5):3059. DOI: https://doi.org/10.1093/jjco/27.5.305. 